\title{
Apoptosis-inducing effect of myxoma virus on human neuroglioma cell lines
}

\author{
QIU-SHENG ZHANG ${ }^{1,2}$, MENG ZHANG $^{2}$, XIAN-JIAN HUANG ${ }^{2}$, XIAO-JIA LIU ${ }^{2}$ and WEI-PING LI ${ }^{2}$ \\ ${ }^{1}$ Department of Neurosurgery, Shenzhen Clinical College Affiliated to Anhui Medical University, Shenzhen, \\ Guandong 518000; ${ }^{2}$ Department of Neurosurgery, Shenzhen 2nd People's Hospital, Shenzhen, Guangdong 508035, P.R. China
}

Received December 16, 2015; Accepted December 23, 2017

DOI: $10.3892 /$ etm.2017.4487

\begin{abstract}
The purpose of this study was to further evaluate the role of myxoma virus (MYXV) as an oncolytic agent against experimental human gliomas in vitro, and analyze the effect of MYXV on malignant glioma cells at different incubation periods and infected at different multiplicities of infection. Neuroglioma cell lines U251 and A172 were cultured with various infective doses of myxoma virus at different time points (0-3 days) and cellular survival rates were evaluated using an MTT assay. Cell viability and cell death rates were assessed using Annexin V/propidium iodide and applying flow cytometry. Furthermore, the expression levels of phosphorylated AKT (p-AKT) in malignant gliomas were detected by western blot analysis to investigate the possible cell signaling targets in the pathway. MYXV exhibited a dose and time-dependent cytotoxic effect on neuroglioma cells, and there was increased expression of $\mathrm{p}-\mathrm{AKT}$ in malignant gliomas. The present study confirms that MYXV induces oncolysis of malignant gliomas through regulating the activation of AKT. As such, MYXV is a potential therapeutic agent against human malignant gliomas.
\end{abstract}

\section{Introduction}

Gliomas occur in ectodermal tissue and are characterized by aggressive proliferation and expansion into surrounding brain tissue. They reportedly account for $\sim 50 \%$ of the incidence rate of neuroepithelial tumors (1). Malignant gliomas are invasive and rapidly growing tumors, which are refractory to available treatments, so they continue to be a major therapeutic challenge. Long-term survivors are rare and the median survival of patients with malignant gliomas is $\sim 1$ year, which has not changed notably in the last 30 years (2). In recent years, glioma therapy has advanced substantially, especially due to molecular

Correspondence to: Dr Wei-Ping Li, Department of Neurosurgery, Shenzhen 2nd People's Hospital, 3002 West Sungang Road, Shenzhen, Guangdong 508035, P.R. China

E-mail: liwpkxy@126.com

Key words: myxoma virus, malignant gliomas, phosphorylated AKT, cytotoxicity, apoptosis biology research. Gene and viral therapies are gradually being developed, with several previous studies and a small number of pre-clinical research reports focused on this area (3-5).

Myxoma virus (MYXV) is a type of poxvirus and has a large double-stranded DNA genome that allows for the potential insertion of large $(25 \mathrm{~kb})$, therapeutically relevant, eukaryotic genes (6). MYXV is a rabbit-specific virus and brings about a lethal disease in the European rabbit $(7,8)$. However, it is an oncolytic virus that is non-pathogenic for all other vertebrate species tested including humans. Despite the extremely narrow species host range, MYXV can effectively infect certain non-rabbit cells in vitro, including primary murine cells with genetic defects of the interferon response, and a variety of human tumor cells in vitro experiments (9-12). It has been shown in previous studies that MYXV exerts an effective and oncolytic potential against human malignant gliomas $(8,9)$. The mechanism underlying the progress of this oncolysis is not yet fully understood. However, there is increasing evidence that the activity of AKT plays a central role in modulating MYXV-mediated oncolysis of diverse tumor cells $(13,14)$.

In this study, MYXV was shown to affect cell viability and induce cell apoptosis, resulting in a dose- and time-dependent cytotoxic effect on neuroglioma cells. The phosphorylation of AKT in the neuroglioma cell lines was induced by MYXV, indicating that MYXV induced oncolysis of malignant gliomas through regulating the activation of AKT. These results suggest that MYXV could have therapeutic value in the treatment of malignant gliomas.

\section{Materials and methods}

Cell cultures. The human neuroglioma cells lines U251 (TCHu 58) and A172 (TCHu171) obtained from the Type Culture Collection of the Chinese Academy of Sciences (Shanghai, China) and were grown in DMEM/F12 (Hyclone, Beijing, China) containing $10 \%$ fetal bovine serum (FBS; Hyclone) at $37^{\circ} \mathrm{C}$ in a humidified $5 \% \mathrm{CO}_{2}$ incubator. All cells were passaged until they reached $80 \%$ confluence, harvested by trypsin treatment, and replaced in the medium. Each cell line was tested routinely for mycoplasma contamination.

Virus and cell infection. Parental myxoma virus (Lausanne strain) was obtained from Grant McFadden at the University 
of Florida (Gainsville, FL, USA) and was amplified in BGMK cells as previously described (15). In brief, BGMK cells were infected with myxoma virus and then harvested following $72 \mathrm{~h}$. These cells were lysed through filtration, and then the supernatant was clarified via a $36 \%$ sucrose pad. Pellets in the supernatant were eliminated by discontinuous $(40 / 36 / 32 / 28 / 24)$ percent sucrose gradient and viral virions were extracted from the $40 / 36 \%$ interface. A derivative of myxoma virus, designated vMyxGFP (16), was prepared and titrated on BGMK cells as described previously (15). Inactive myxoma virus was prepared by irradiating vMyxGFP with UV light for $3 \mathrm{~h}$. U251 and A172 cells were infected with vMyxGFP or inactive myxoma virus at multiplicities of infection (MOIs) of 0, 0.1, $0.5,1,5$ and 10 for $1 \mathrm{~h}$ at $37^{\circ} \mathrm{C}$, and then the cells were washed with phosphate-buffered saline (PBS) and cultured with fresh medium for subsequent experiments.

MTT assay. MTT assay was used to detect the viability of cells infected with 6 gradients $(0,0.1,0.5,1,5$ and 10) of multiplicity of infection (MOI) of MYXV in U251 and A172 cells. The MOI 5, with 40-60\% cell lethality was then used in the subsequent experiments. In brief, cells were plated by using fresh DMEM/F12 medium (Hyclone) supplemented with $10 \%$ FBS in 96-well plates at a density of 5,000 cells per well. Following overnight cultivation at $37^{\circ} \mathrm{C}$, cells were infected with MYXV for 1, 2 or 3 days. After incubating with an additional $25 \mu \mathrm{l}$ MTT solution $(5 \mathrm{mg} / \mathrm{ml}$ ) for $4 \mathrm{~h}$ under $5 \%$ $\mathrm{CO}_{2}$ at $37^{\circ} \mathrm{C}, 100 \mu \mathrm{l}$ dimethyl sulfoxide (DMSO) was added and the container was agitated for $5 \mathrm{~min}$. The precipitated formazan was dissolved, and detected using a microplate reader at $595 \mathrm{~nm}$.

Cell death assay. Apoptotic and necrotic cell populations were evaluated using Annexin V-FITC/propidium iodide (PI). $\mathrm{U} 251$ and A172 cells were seeded by a density of $5 \times 10^{4}$ cells in flasks and incubated under $5 \% \mathrm{CO}_{2}$ at $37^{\circ} \mathrm{C}$ in an incubator until they reached $80 \%$ confluence. Cells treated with MYXV at MOIs of 0,1 and 5 were harvested at 0,1 and 2 days post-infection, then washed with PBS twice, centrifuged at $805 \times \mathrm{g}$ for $5 \mathrm{~min}$ and resuspended in $1 \mathrm{ml}$ binding buffer. Following this, cells were stained with $5 \mu \mathrm{l}$ PI and examined using an Annexin V-FITC apoptosis detection kit (Cell Signaling Technology, Inc., Beverly, MA, USA). Cells were incubated at room temperature in a dark environment for $15 \mathrm{~min}$. The percentage of cell death was assessed by flow cytometry (FCM) using CellQuest software (BD Biosciences, San Jose, CA, USA).

Western blot analysis. To study the effect of MYXV on phosphorylated AKT (p-AKT) in neuroglioma cells, total AKT and p-AKT expression was evaluated in U251 and A172 cells by western blot analysis. Neuroglioma cells were infected with $5 \mathrm{MOI}$ of MYXV, and untreated cells were set as a negative control. After being infected for 0,1 or 2 days, whole proteins were extracted from cell lysates with RIPA lysis buffer (Beyotime Institute of Biotechnology, Shanghai, China; $50 \mathrm{nM}$ Tris, $\mathrm{pH}$ 7.4, $150 \mathrm{mM} \mathrm{NaCl,} \mathrm{1 \%} \mathrm{Triton} \mathrm{X-100,}$ $1 \%$ sodium deoxycholate, $0.1 \%$ SDS, 0.05 mM EDTA), separated by $10 \%$ SDS-PAGE and transferred onto nitrocellulose membranes (Hoffman-La Roche, AG, Basel, Switzerland).

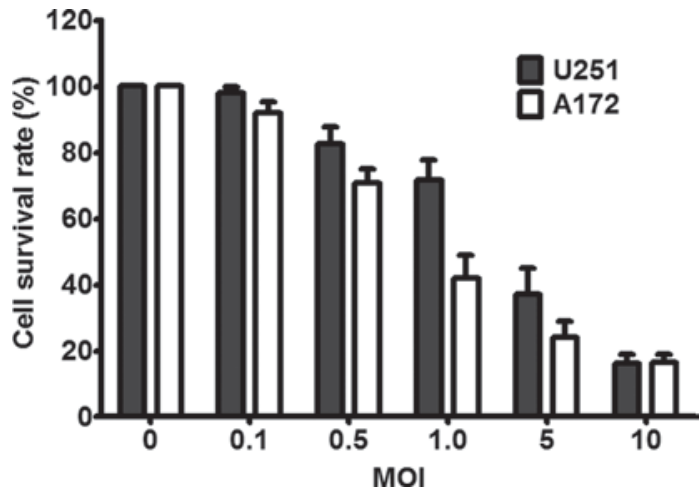

Figure 1. MYXV induces cell death in a dose-dependent manner. U251 and A172 cells were infected with different MOIs of MYXV and their survival rate was detected by MTT assay three days later. MYXV, myxoma virus; MOI, multiplicity of infection.

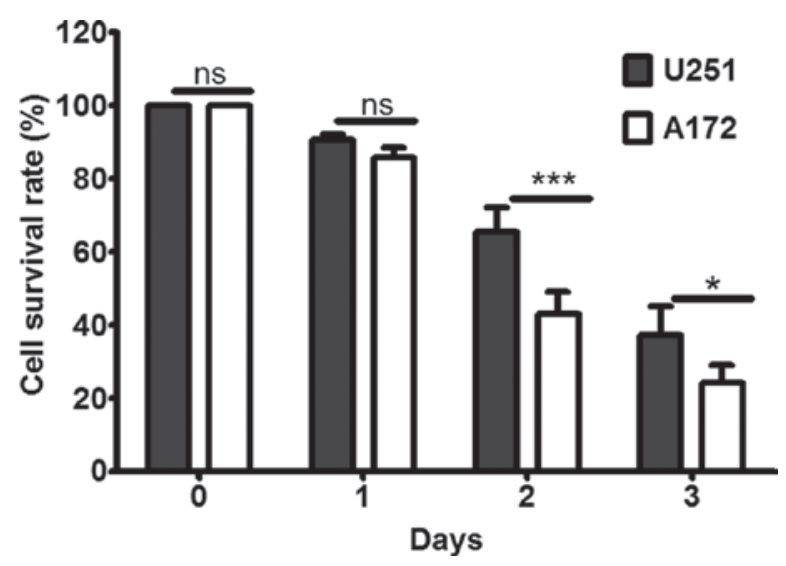

Figure 2. MYXV induces cell death in a time-dependent manner. U251 and A172 cells were infected with MYXV (MOI=5), for periods of 1, 2 or 3 days, after which the cell survival rate was detected by MTT. ${ }^{* * *} \mathrm{P}<0.0012$ days post-infection of $\mathrm{U} 251$ cells vs. A172 cells, ${ }^{*} \mathrm{P}<0.053$ days post-infection of U251 cells vs. A172 cells. Ns, no statistical significance; MYXV, myxoma virus; MOI, multiplicity of infection.

The membranes were blocked with $5 \%$ skimmed milk in Tris-buffered saline, then incubated with primary polyclonal antibodies anti-AKT (pan; catalogue no. C67E7) at a dilution of 1:1,000 and monoclonal anti-p-AKT (Ser-473; catalogue no. D9E) XP at a dilution of $1: 1,000$ at $4^{\circ} \mathrm{C}$ overnight, both purchased from Cell Signaling Technology, Inc (Beverly, MA, USA). Afterwards, the membranes were incubated for $1 \mathrm{~h}$ at room temperature with horseradish peroxidase-conjugated secondary antibody (1:10,000; catalogue no. HAF017; R\&D Systems; Bio-Techne, Minneapolis, MN, USA). The bands were visualized using an ECL Western Blotting Kit (BioVision, Inc., Milpitas, CA, USA) and quantified by Quantity One version 4.6.2 software (Bio-Rad Laboratories, Inc., Hercules, CA, USA).

Statistical analysis. All experiments were repeated a minimum of three times. All data are presented as mean \pm standard deviation wherever applicable. GraphPad Prism version 5 software was used to perform statistical analysis. We used a Student's t-test and two-way analysis of variance (ANOVA) to determine significance. $\mathrm{P}<0.05$ was considered to represent statistically significant differences. 

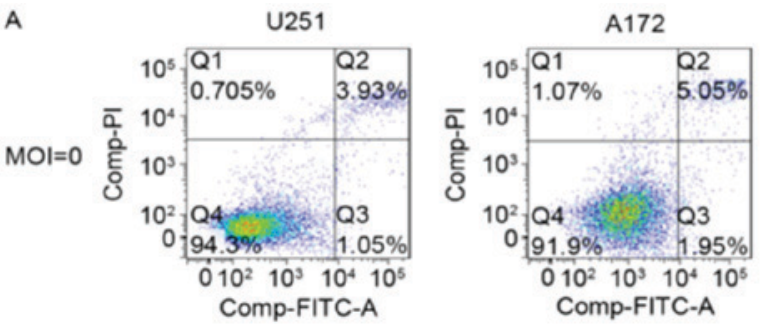

B

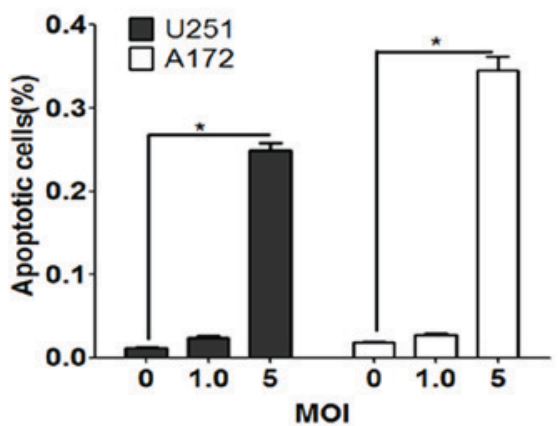

MOI=1
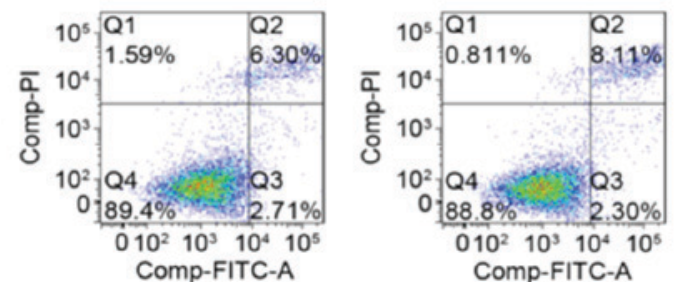

MOI
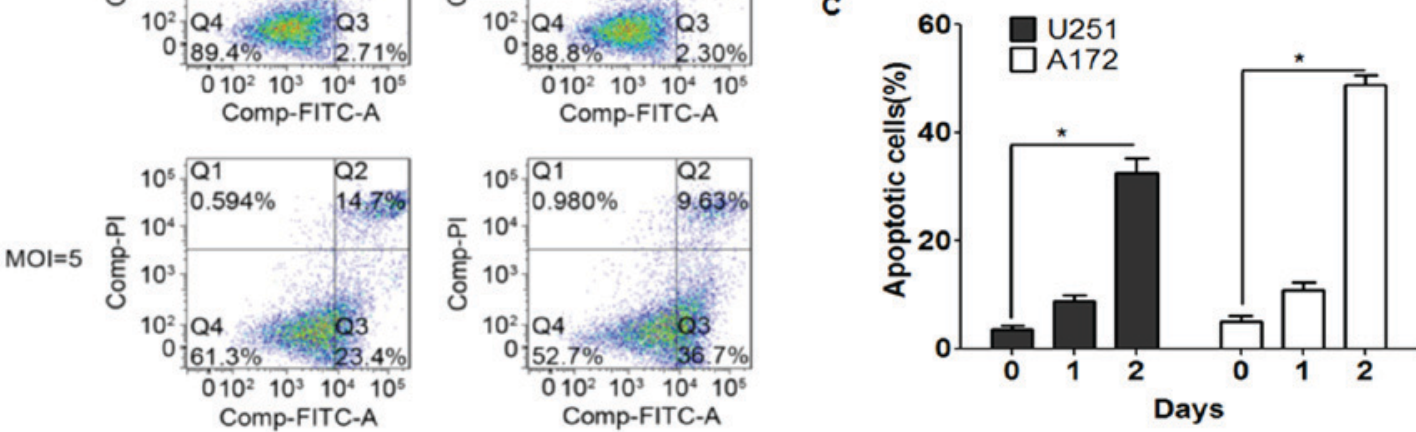

Figure 3. Effect of MYXV on cell death in different incubation periods. (A) Representative dot plot of cell population distribution stained for Annexin V-FITC/PI at MOIs of 0,1 and 5. (B) Percentage of apoptotic cells in U251 and A172 when treated with 5 MOI MYXV for 0,1 and 2 days. "P<0.05 vs. 0 days. MYXV, myxoma virus; PI, propidium iodide; MOI, multiplicity of infection.

A

Aay (s)

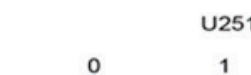

251
1

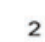

p-AKT
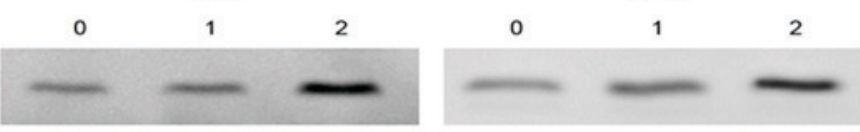

AKT
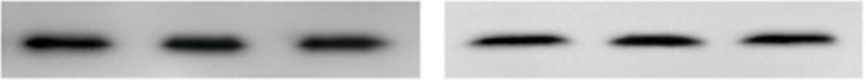

B
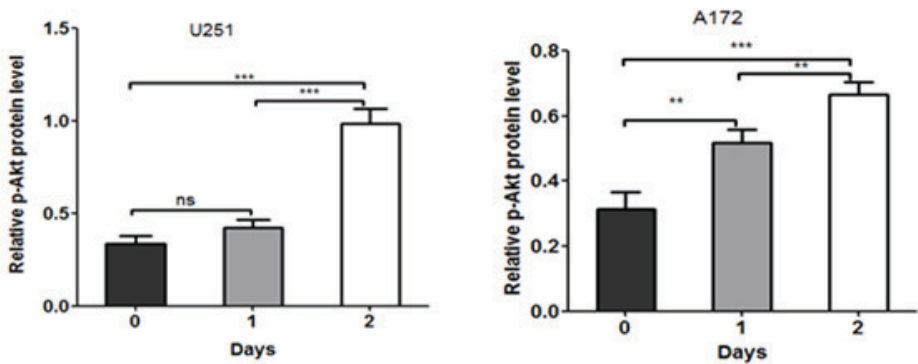

Figure 4. Effect of MYXV on p-AKT activity in U251 and A172 cells. (A) Western blot analysis was used to detect total AKT and p-AKT in U251 and A172 cells 0,1 and 2 days post-MYXV infection. (B) Relative p-AKT protein level in U251 and A172 cells after 0,1 and 2 days. ${ }^{* * *} \mathrm{P}<0.0011$ or 2 days post-infection vs. 0 days, ${ }^{* *} \mathrm{P}<0.012$ vs. 1 days post-infection of A172 cells or 1 vs. 0 days post-infection of A172 cells. Ns, no statistical significance; MYXV, myxoma virus; p-AKT, phosphorylated AKT.

\section{Results}

MYXV affected cell viability in a dose-dependent manner. Different infective doses of cell lethality were investigated and similar results were found in two human malignant glioma cell lines (U251 and A172). As shown in Fig. 1, both U251 and A172 cells were susceptible to infection by MYXV, which was consistent with a previous study (16). Furthermore, $>80 \%$ of the U251 and A172 cells were killed by MYXV at an MOI of 10. When the MOI was 5, both U251 and A172 cell lethality was between 40 and $60 \%$, which was determined to be the most suitable infection status for the present study. In addition, the cell viabilities of the U251 and A172 cells that did not receive myxoma virus were used as negative control for all experiments in the present study. Based on this result, an MOI of 5 was used in the subsequent experiments. 
MYXV affected cell viability in a time-dependent manner. The effect of different incubation periods of MYXV on cell death was determined by MTT assay. Neuroglioma cells were infected with MYXV at MOI of 5. After incubation periods of $0,1,2$ and 3 days, the survival rates of U251 and A172 were detected by MTT. Cell survival rates were reduced as the length of the incubation period increased, and A172 cells were more sensitive to MYXV than U251 cells $(\mathrm{P}<0.05$; Fig. 2).

MYXV induced cell apoptosis in a time- and dose-dependent manner. FCM was conducted to detect the effect of MYXV on cell apoptosis. Neuroglioma cells were infected with MYXV at different MOIs (0, 1 and 5). As shown in Fig. 3A and $\mathrm{B}$, compared with MOI 0 , the proportion of apoptotic cells in U251 and A172 cells increased at MOI 5, which indicated that both $\mathrm{U} 251$ and A174 cells were susceptible to being killed by MYXV in a dose-dependent manner $(\mathrm{P}<0.05$; Fig. 3A and B). Furthermore, the percentage of apoptotic cells in U251 and A172 after treatment with 5 MOI MYXV for 0, 1 and 2 days were calculated (Fig. 3C). With the increase of time post-infection, cell apoptosis rate of U251 and A172 cells also increased compared to day 0 ( $\mathrm{P}<0.05$; Fig. $3 \mathrm{C})$, which suggested that MYXV induced cell apoptosis in a time-dependent manner.

Effect of MYXV on $p$-AKT activity. The levels of p-AKT are directly involved in the susceptibility of tumor cells to infection with MYXV $(9,17)$. To examine whether similar effects are observed in U251 and A172 cell lines, western blot analysis was used to detect the expression levels of total AKT and p-AKT in U251 and A172 cells that had been infected by MYXV at MOI 5 for 0,1 and 2 days. Consistent with previous studies, MYXV induced the phosphorylation of AKT in both U251 and A172 cell lines in a time-dependent manner (Fig. 4). Then a densitometric analysis was performed on these results by Quantity One version 4.6.2 software. According to the densitometric analysis, in U251 cells, there was a significant increase in the expression levels of $\mathrm{p}$-AKT on day 2 compared with day $0(\mathrm{P}<0.05$; Fig. $4 \mathrm{~B})$ and in $\mathrm{A} 172$ cells, there was a significant increase in $\mathrm{p}$-AKT expression levels on day 1 and 2 compared to day $0(\mathrm{P}<0.05 ;$ Fig. 4B).

\section{Discussion}

Malignant gliomas are the most common and aggressive primary central nervous system tumor in humans. Numerous types of conventional pro-apoptotic therapies have been applied to resistant malignant gliomas, such as radiotherapy, chemotherapy and adjuvant therapies, but these have not been effective in killing glioma cells $(18,19)$. It is therefore crucial to develop an original approach for malignant glioma therapy. Oncolytic virotherapy, a novel and promising cancer therapeutic strategy, is reported to be more effective and have fewer side effects than conventional cancer therapies (20-22), and has previously been investigated as a treatment for gliomas (23). Candidate oncolytic viruses should produce few side-effects, be non-pathogenic and exhibit selective anti-tumor activities.

MYXV belongs to the poxviridae family. It is a rabbit-specific virus and exhibits restricted pathogenicity for all other vertebrate species, including humans $(24,25)$. Despite its limited host range specificity, MYXV has been shown to selectively infect diverse forms of human tumor cells, including glioma cells (8). In the present study, MYXV acted as a vital factor that affected cell survival rates. In addition, MYXV exerted such effects in a dose and time-dependent manner. The results also indicated that MYXV contributed to apoptosis in human neuroglioma cell lines U251 and A172 in a dose and time-dependent manner.

Notably, MYXV exerts a selective tropism for tumor cells with elevated levels of p-AKT (8). AKT, also known as protein kinase $\mathrm{B}$, is a serine/threonine protein kinase which plays an important role in cell survival and apoptosis (17). AKT phosphorylates a series of proteins and inhibits apoptosis via a number of mechanisms (26). It has been confirmed that the majority of both malignant and recurrent glioma cells exhibit PTEN gene inactivation or deletion, which increases AKT activity and results in cell proliferation and inhibition of apoptosis. Data from the current study indicated that the expression levels of p-AKT significantly increased in MYXV-infected $\mathrm{U} 251$ and A172 cell lines compared with cell lines receiving an inactive virus.

Furthermore, the present results verified the stated association between the activation of AKT and MYXV-mediated oncolysis in vitro. This highlights the potential oncolytic function of MYXV on human glioma cells and provides a promising therapeutic target for human malignant glioma tumors. Further studies are required to verify whether MYXV can promote oncolysis through modulating the levels of activated AKT within other types of tumor cell in vitro, which could make MYXV a key factor in improving the outcome of treatment for various cancer types.

\section{Acknowledgements}

This work was supported by Shenzhen InternationalCooperation Research Funding (grant no. GJHZ20120614154914623) and Shenzhen Key Laboratory of Neurosurgery (grant no. ZDSYS20140509173142601).

\section{References}

1. Laws ER: Management of (malignant) intracranial gliomas. Practical Handbook of Neurosurgery: 549-558, 2009.

2. Scott JN, Rewcastle NB, Brasher PM, Fulton D, MacKinnon JA, Hamilton M, Cairncross JG and Forsyth P: Which glioblastoma multiforme patient will become a long-term survivor? A population-based study. Ann Neurol 46: 183-188, 1999.

3. Wilcox ME, Yang W, Senger D, Rewcastle NB, Morris DG Brasher PM, Shi ZQ, Johnston RN, Nishikawa S, Lee PW and Forsyth PA: Reovirus as an oncolytic agent against experimental human malignant gliomas. J Natl Cancer Inst 93: 903-912, 2001.

4. Csatary LK, Gosztonyi G, Szeberenyi J, Fabian Z, Liszka V, Bodey B and Csatary CM: MTH-68/H oncolytic viral treatment in human high-grade gliomas. J Neurooncol 67: 83-93, 2004.

5. Freeman AI, Gomori JM, Linetsky E, Zakay-Rones Z, Panet A, Libson E, Irving CS, Galun E and Siegal T: Phase I/II trial of intravenous OV001 oncolytic virus in resistant glioblastoma multiforme (GBM). J Clin Oncol 22: 1515, 2004

6. Stanford MM and McFadden G: Myxoma virus and oncolytic virotherapy: A new biologic weapon in the war against cancer. Expert Opin Biol Ther 7: 1415-1425, 2007.

7. Kirn DH and Thorne SH: Targeted and armed oncolytic poxviruses: A novel multi-mechanistic therapeutic class for cancer. Nat Rev Cancer 9: 64-71, 2009. 
8. Lun X, Yang W, Alain T, Shi ZQ, Muzik H, Barrett JW, McFadden G, Bell J, Hamilton MG, Senger DL and Forsyth PA: Myxoma virus is a novel oncolytic virus with significant antitumor activity against experimental human gliomas. Cancer Res 65: 9982-9990, 2005

9. Lun XQ, Zhou H, Alain T, Sun B, Wang L, Barrett JW, Stanford MM, McFadden G, Bell J, Senger DL and Forsyth PA: Targeting human medulloblastoma: Oncolytic virotherapy with myxoma virus is enhanced by rapamycin. Cancer Res 67: 8818-8827, 2007

10. Almansour NM, Pirogova E, Coloe PJ, Cosic I and Istivan TS: A bioactive peptide analogue for myxoma virus protein with a targeted cytotoxicity for human skin cancer in vitro. J Biomed Sci 19: 65, 2012.

11. Coffey MC, Strong JE, Forsyth PA and Lee PW: Reovirus therapy of tumors with activated ras pathway. Science 282: 1332-1334, 1998.

12. Norman KL, Hirasawa K, Yang AD, Shields MA and Lee PW: Reovirus oncolysis: The Ras/RalGEF/p38 pathway dictates host cell permissiveness to reovirus infection. Proc Natl Acad Sci USA 101: 11099-11104, 2004

13. Werden SJ and McFadden G: Pharmacological manipulation of the Akt signaling pathway regulates myxoma virus replication and tropism in human cancer cells. J Virol 84: 3287-3302, 2010.

14. Correa RJ, Komar M, Tong JG, Sivapragasam M, Rahman MM, McFadden G, Dimattia GE and Shepherd TG: Myxoma virus-mediated oncolysis of ascites-derived human ovarian cancer cells and spheroids is impacted by differential AKT activity. Gynecol Oncol 125: 441-450, 2012.

15. Smallwood SE, Rahman MM, Smith DW and McFadden G: Myxoma virus: Propagation, purification, quantification and storage. Curr Protoc Microbiol Chapter 14: Unit: 14A. 1, 2010.

16. Johnston JB, Barrett JW, Chang W, Chung CS, Zeng W, Masters J, Mann M, Wang F, Cao J and McFadden G: Role of the serine-threonine kinase PAK-1 in myxoma virus replication. J Virol 77: 5877-5888, 2003.
17. Wang G, Barrett JW, Stanford M, Werden SJ, Johnston JB, Gao X, Sun M, Cheng JQ and McFadden G: Infection of human cancer cells with myxoma virus requires Akt activation via interaction with a viral ankyrin-repeat host range factor. Proc Natl Acad Sci USA 103: 4640-4645, 2006.

18. Hentschel SJ and Lang FF: Current surgical management of glioblastoma. Cancer J 9: 113-125, 2003

19. Stupp R, Mason WP, van den Bent MJ, Weller M, Fisher B, Taphoorn MJ, Belanger K, Brandes AA, Marosi C, Bogdahn U, et al: Radiotherapy plus concomitant and adjuvant temozolomide for glioblastoma. N Engl J Med 352: 987-996, 2005.

20. Thorne SH and Kirn DH: Future directions for the field of oncolytic virotherapy: A perspective on the use of vaccinia virus. Expert Opin Biol Ther 4: 1307-1321, 2004.

21. Shah AC, Benos D, Gillespie GY and Markert JM: Oncolytic viruses: Clinical applications as vectors for the treatment of malignant gliomas. J Neurooncol 65: 203-226, 2003

22. Norman KL, Farassati F and Lee PW: Oncolytic viruses and cancer therapy. Cytokine Growth Factor Rev 12: 271-282, 2001.

23. Hu CW, Yin GF, Wang XR, Ren BW, Zhang WG, Bai QL, Lv YM, Li WL and Zhao WQ: IL-24 induces apoptosis via upregulation of RNA-activated protein kinase and enhances temozolomide-induced apoptosis in glioma cells. Oncol Res 22: 159-165, 2014.

24. Górski J, Mizak B and Chrobocińska M: Control of rabbit myxomatosis in poland. Rev Sci Tech 13: 869-879, 1994.

25. Rivers TM and Ward SM: Infectious myxomatosis of rabbits: Preparation of elementary bodies and studies of serologically active materials associated with the disease. J Exp Med 66: 1-14, 1937.

26. Barrett JW, Alston LR, Wang F, Stanford MM, Gilbert PA, Gao X, Jimenez J, Villeneuve D, Forsyth P and McFadden G: Identification of host range mutants of myxoma virus with altered oncolytic potential in human glioma cells. J Neurovirol 13: 549-560, 2007. 